\title{
Protective role of propolis against histological alterations in renal tissue induced by gold nanoparticles
}

\author{
Mansour I. Almansour ${ }^{\mathrm{a}}$, Mosaid A. Alferah ${ }^{\mathrm{b}}$, Bashir M. Jarrar ${ }^{\mathrm{c}, *}$ \\ a Department of Zoology, College of Science, King Saud University, Saudi Arabia \\ b Biology Department, College of Science-Onizah, Qassim University, Saudi Arabia \\ c Department of Biological Sciences, College of Science, Jerash University, Jordan \\ *Corresponding author, e-mail: bashirjarrar@yahoo.com
}

Received 2 Feb 2015

Accepted 11 Sep 2016

\begin{abstract}
Male albino Wistar rats were exposed to $10 \mathrm{~nm}$ gold nanoparticles at a dose of $2000 \mu \mathrm{g} / \mathrm{kg}$ together with or without propolis $(50 \mathrm{mg} / \mathrm{kg}$ ) for 15 consecutive days. Fresh renal biopsies from of all investigated rats were cut rapidly, fixed in neutral buffered formalin, subjected to histological processing, and examined for microanatomical alterations. Propolis gave full protection against glomerular congestion and renal tubule hyaline casts. It demonstrated partial amelioration against glomerular capillary dilatation, tubular cloudy swelling, necrosis, and degeneration together with interstitial blood capillaries dilatation and haemorrhage induced by nanoparticle toxicity. On the other hand, propolis showed no protective effect against renal cells pyknosis, karyolysis, apoptosis and renal hydropic degeneration together with collecting tubules atrophy and degeneration induced by the nanoparticles. Thus propolis can augment the antioxidant defence against the severity of some alterations in the renal tissues. This ameliorative role might be related to the antioxidants content of propolis that protect the renal tissues from free radicals and oxidative stress.
\end{abstract}

KEYWORDS: nanotoxicity, kidney, antioxidant, glomerulus, renal tubules

\section{INTRODUCTION}

Gold nanoparticles (GNPs) have promise in multiple diagnostic and therapeutic application together with a variety of industrial purposes ${ }^{1,2}$. These fine particles have unique properties that make them biologically active with affinity to accumulate in the vital organs mainly kidney liver and spleen ${ }^{3-5}$. Previous research work demonstrated the potential toxic effect of GNPs due to their size, surface area, shape, and charge where smaller particles are more toxic than the larger ones ${ }^{6-8}$. GNPs demonstrated oxidative stress and macromolecules interaction that could result in histocytotoxicity ${ }^{9-11}$.

Some research works showed that GNPs with a size of of $5-20 \mathrm{~nm}$ were more toxic and had long time accumulation than the larger ones in the vital organs including the kidney ${ }^{12-14}$. The kidneys receive high blood flow and have high exposure to small GNPs with long circulating residue than the larger ones $9,15,16$.

Propolis is a bee glue consists of natural resinous substances collected from plants and able to keep and protect the hives from invaders and envi- ronmental harsh conditions ${ }^{17,18}$. Propolis is well known for its antioxidant properties and has been used for long time as therapeutic and supportive agent ${ }^{19,20}$. In addition, this natural crude is being used widely in pharmaceutical industry and cosmetic applications due to its antioxidative properties and abilities to prevent lipid peroxidation ${ }^{21,22}$. Some studies reported propolis to have role in a blood capillaries strengthening and tissue regeneration $^{23}$. Furthermore, propolis was found to have protective role from some chemicals and environmental toxic substances such as carbon tetrachloride, acetaminophen, and inorganic toxicity ${ }^{24-26}$. The aim of the present project is to determine whether propolis can protect the renal tissues from the histological alterations induced by GNPs toxicity.

\section{MATERIALS AND METHODS}

\section{Experimental animals}

Forty male adult albino Wistar rats weighing 210$230 \mathrm{~g}$ were obtained from the animal house (College of Pharmacy, King Saud University, Saudi Arabia). The rats were randomly assigned and separately 
caged into three test groups and a control one (10 rats each) with access to food and water ad libitum.

\section{Gold nanoparticles}

Spherical colloidal monodisperse GNPs $(10 \mathrm{~nm})$ suspended in $0.1 \mathrm{mM}$ phosphate-buffered saline, were purchased from Sigma-Aldrich (USA), with the following physicochemical characterization: $5.98 \times 10^{12}$ nanoparticles $/ \mathrm{ml}$, concentration of $1.01 \times 10^{8} \mathrm{M}^{-1} \mathrm{~cm}^{-1}$, surface area of each particle of $3.16 \times 10^{-12} \mathrm{~cm}^{2}$, reactant free, absorption at $\sim 520 \mathrm{~nm}$.

\section{Propolis}

Commercial water soluble propolis crude in the form of capsules (1000 mg) manufactured by Marnys Spanish Company (Spain) and imported by Saudi Arabian Dug Store Ltd (Saudi Arabia) was used. Its active ingredients were identified by the quality control of the manufacturer. The analysis indicated the following contents. Phenolic acids (caffeic acid, tocopherol, sinapic acid, cinnamic acid, coumaric acid, and ferulic acid) and flavonoid compounds (quercetin, kaempferol, rutin, and apigenin) together with amino acids and vitamins.

For the use of propolis in the present work, the capsules content was dissolved immediately before use in sterile distilled water. The rats were subjected to propolis in a daily single dose for 15 consecutive days.

\section{Experimental protocol}

The rats were exposed to GNPs together with or without propolis as follows:

Group I: received neither GNPs nor propolis but a single intraperitoneal injection of $100 \mu \mathrm{l}$ of the nanoparticles vehicle in deionized water for consecutive 15 days.

Group II: received a daily intraperitoneal injection of $100 \mu \mathrm{l}$ GNPs of size $10 \mathrm{~nm}$ at a dose of $2000 \mu \mathrm{g} / \mathrm{kg}$ for consecutive 15 days.

Group III: received a daily intraperitoneal injection of $100 \mu \mathrm{l}$ GNPs of size $10 \mathrm{~nm}$ at a dose of $2000 \mu \mathrm{g} / \mathrm{kg}$, before being exposed to single oral dose of propolis $(50 \mathrm{mg} / \mathrm{kg}$ ) for consecutive 15 days.

Group IV: received a daily oral dose of propolis (50 $\mathrm{mg} / \mathrm{kg}$ ) for consecutive 15 days.

\section{Sample preparation}

All members of all groups were euthanized by cervical dislocation after 15 days of treatment. Fresh biopsies from the right kidney of each rat of all groups were cut rapidly, fixed in neutral buffered formalin, dehydrated with ascending grades of ethanol, cleared in 2 changes of xylene, impregnated with 2 changes of molten paraffin wax, then embedded and blocked out. Paraffin sections (4$5 \mu \mathrm{m}$ ) of the control and GNPs treated rats were stained according to Jarrar and Taib ${ }^{27}$ with haematoxylin and eosin (H\&E) stain and other special stains.

\section{Microscopic examination}

Histological sections of all rats under study were examined using Olympus light microscope while the digital photography was carried out by using Olympus optical microscope with digital camera.

All animals were handled and the experiments were conducted in accordance with the protocols approved by King Saud University ethical committee. The doses and route of administration were carried out according to the protocols from previous studies and confirmed data from the literature.

\section{RESULTS}

The kidneys of the control rats showed normal histological picture of glomerular, tubular and interstitial components of both the cortex and the medulla (Fig. 1a). The kidneys of rats exposed to GNPs with or without propolis, however, showed the following glomerular, tubular, and interstitial histological alterations:

Glomerular congestion: the rats exposed to $10 \mathrm{~nm}$ GNPs for consecutive 15 days demonstrated marked occasional glomerular capillary congestion (Fig. 1b). This glomerular damage was not seen in the kidneys of rats exposed to GNPs plus propolis.

Glomerular capillary dilatation: Ballooned glomerular capillaries were detected in the renal tissues of rats exposed to GNPs (Fig. 1c). The kidneys of rats exposed to GNPs plus propolis demonstrated less glomerular capillaries dilatation in comparison to those subjected to GNPs only.

Renal tubules cloudy swelling: all rats subjected to GNPs treatment showed renal tubules epithelial lining cloudy swelling. This abnormality was more prominent in the epithelial lining of the proximal convoluted tubules than the distal ones (Fig. 1d). This damage was less prominent in the renal tubules exposed to both GNPs plus propolis.

Renal tubules hydropic degeneration: Vacuolization of the renal cells was seen in the renal tubules of rats received $10 \mathrm{~nm}$ for 15 days (Fig. 1e). Treatment with propolis for 15 days showed little amelioration, if any, in this renal tissue damage. 

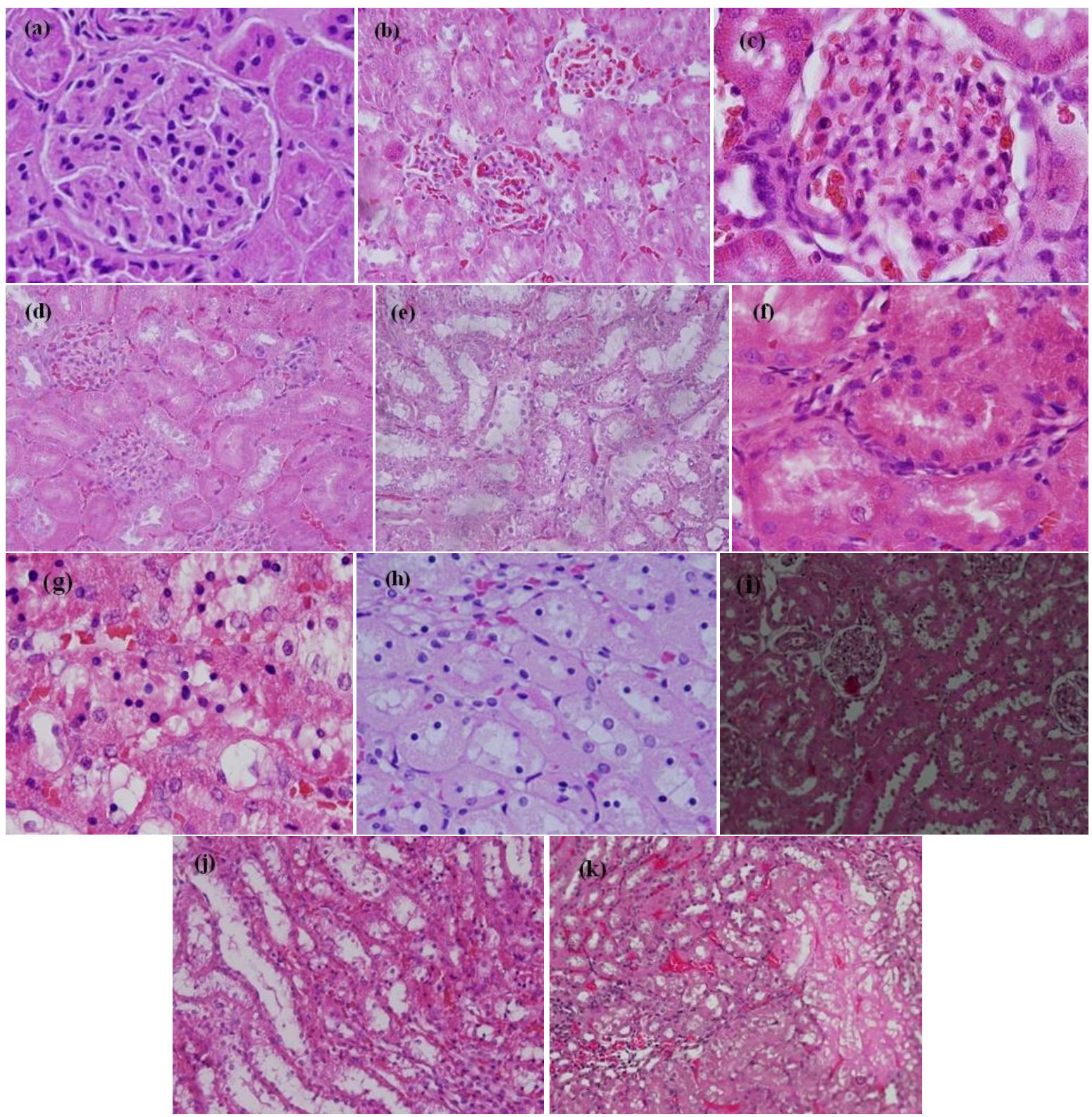

Fig. 1 Light micrographs demonstrating: (a) control kidney with normal glomerular and tubular structures, (b) glomerular congestion, (c) glomerular capillary dilatation, (d) renal tubule cloudy swelling, (e) hydropic degeneration, (f) cytoplasmic hyaline droplets and hyaline casts, (g) renal cells nuclear alterations, (h) prominent tubular necrosis, (i) renal tubular degeneration, (j) collecting tubules degeneration, (k) interstitial capillary dilatation and haemorrhage. H\&E stain.

Hyaline droplets and hyaline casts: rats received GNPs for 15 days demonstrated cytoplasmic hyaline droplets in the epithelium of the renal tubules (Fig. 1f). Moreover, occasional hyaline casts were also seen in the lumen of some distal convoluted renal tubules. The hyaline casts of the renal tubules were not seen in the renal tissues of rats exposed to GNPs plus propolis in comparison with rats subjected to GNPs only.

Renal tubules nuclear alterations: some cells lining the proximal convoluted tubules of rats exposed to GNPs demonstrated anisokaryosis, pyknosis, karyorrhexis, karyolysis and apoptosis while the nuclei of the distal tubules were less affected (Fig. 1g). No obvious protection from these alterations was seen in the renal tubules of rats subjected 
to GNPs and propolis for consecutive 15 days.

Tubular necrosis: necrosis was observed in the renal tubules of rats exposed to GNPs for 15 days (Fig. 1h). This alteration was more prominent in the cortex than the medulla. The renal tubules of rats subjected to GNPs plus propolis showed less necrosis than rats treated with GNPs only. Occasional cytoplasmic eosinophilia in some necrotic proximal convoluted tubules was also seen in the renal epithelium of rats exposed to GNPs but not in rats subjected to both GNPs plus propolis.

Renal tubules degeneration: swelling cytolysis and thinning or absence of the proximal tubular brush border were also seen in some renal tubules of rats exposed to GNPs only (Fig. 1i). The proximal renal tubules became flattened and were more affected than the distal ones. This damage was less prominent in the kidneys of rats subjected to GNPs plus propolis.

Collecting ducts degeneration: Atrophied collecting ducts were seen in the renal tissues of rats treated with GNPS (Fig. 1j). Propolis failed to protect the collecting ducts of rats subjected to GNPs from this damage.

Intertubular blood capillaries dilatation and haemorrhage: the renal tissues of rats exposed to GNPs only demonstrated intertubular blood capillaries dilatation together with occasional intertubular haemorrhage (Fig. 1k). These alterations were less prominent in the kidneys of rats treated with GNPs plus propolis.

\section{Kidneys of rats exposed to propolis only}

Rats exposed to propolis $(50 \mathrm{mg} / \mathrm{kg}$ ) only for consecutive 15 days demonstrated normal histological picture with no abnormalities in the glomerular, tubular or interstitial constituents in comparison with the renal tissues of the control rats.

\section{DISCUSSION}

Previous studies indicated that GNPs may reveal a high risk potential on the microstructure and function of the kidney ${ }^{28}$. The results of the present work showed that the cortex and the proximal convoluted tubules were more affected than the medulla and the distal tubules, respectively. This may indicate that more GNPs reach the cortex via the blood stream than that would enter the medulla where most of the total renal blood flow enters the cortex.

Our results showed partial amelioration to cloudy swelling and cytoplasmic vacuolating injuries induced by GNPs with propolis subjection. This may indicate that this natural product can reverse partially the change in the ion balance and fluid haemostasis that affects water and ion transport through cell membrane due to GNPs toxicity. Some investigators reported that propolis could repair cellular structure and functions by inhibiting cell membrane free radical formation and combating lipid peroxidation by activation of some antioxidant enzymes ${ }^{29-31}$.

Propolis subjection improved glomerular, intertubular, and interstitial capillary dilatation induced by GNPs. This might indicate potential capability of propolis to suppress glomerular filtration surface and interstitial renal capillary expansion related to its ability to prevent lipid peroxidation by activation of some antioxidant enzymes ${ }^{14,17,29}$. Several studies reported that propolis could protect the mitochondria and cellular macromolecules against oxidative damage ${ }^{32-34}$. In addition, the protective role of propolis against formation of hyaline casts might indicate an ability to ameliorate protein disturbances induced by GNPs (Fig. 2).

On the other hand, the results of the present work showed that propolis was unable to prevent renal cells anisokaryosis, pyknosis, karyorrhexis, karyolysis, and apoptosis (Fig. 2b) together with tubular hydropic degeneration and collecting tubules atrophy towards normal. This might indicate that the oxidative power of propolis cannot compensate for the damage in these tubules that most likely resulted from hydrolytic enzyme leakage induced by GNPs exposure. Pyknosis is a sort of condensation of the chromatin materials in the periphery of the nuclei with irregularity nuclear membranes while karyolysis is characterized by chromatin dissolution of a dying cell ${ }^{35}$. The findings of the present study showed that the induced nuclear alterations by GNPs were seen mainly in the necrotic renal cells. This might indicate that GNPs could induce swelling and lysis of renal cells organelles including the nuclei. Moreover, the failure of propolis to ameliorate the collecting duct atrophy might indicate inability to compensate the hydrostatic pressure induced in these tubules by GNPs.

The findings of the present work are in line with some studies which reported that propolis could protect against the toxicity of several chemical and environmental toxic substances ${ }^{31-33}$. This potential might be related to its pharmacological and biological contents such as flavonoid compounds and phenolic acids. Furthermore, propolis has the ability to activate antioxidant enzymes to suppress cytochrome p-450 enzymes and to reduce lipid peroxidation $^{19,21,30}$. In addition, some investigators 

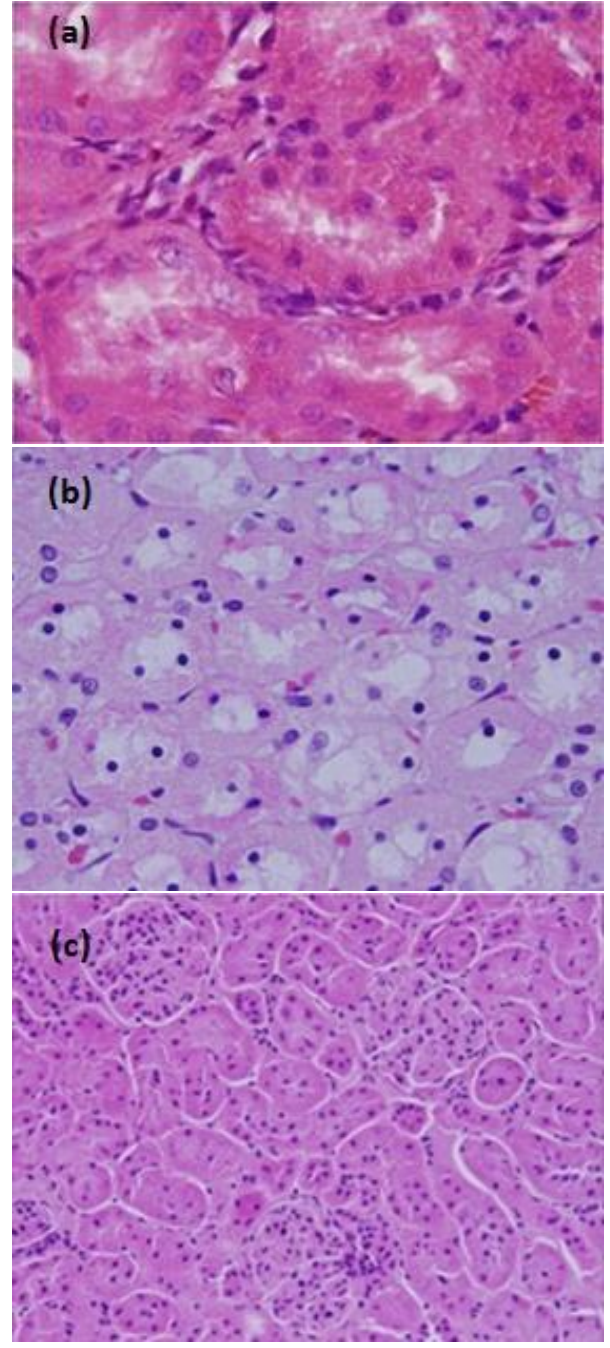

Fig. 2 Light micrographs demonstrating; (a) kidney of rat exposed to GNPs plus propolis showing cytoplasmic hyaline droplets but no hyaline casts in the distal tubules luminae, (b) kidney of rat exposed to GNPs plus propolis showing nuclear alterations almost similar to that seen in subjected to GNPs only, (c) kidney of rat received propolis only demonstrating normal histological structures. H\&E staining.

reported that propolis can inhibit membrane free radical formation and has the capability to protect the mitochondria and cellular macromolecules against oxidative damage ${ }^{31,32}$.

It might be concluded from the findings of the present work that propolis can augment the antioxidant defence against the severity of some alterations in renal tissues induced by GNPs (Fig. 2c). Renal tissues amelioration, strengthening and regeneration by this natural crude against histological damage induced by GNPs might be related to its antioxidant activity. In addition, the present work may provide evidence for propolis therapeutic potential related to its antioxidant ability to protect structure and function of kidney from oxidative stress induced by drugs, toxic substances and pollutants.

Acknowledgements: The authors would like to extend their sincere appreciation to the Deanship of Scientific Research at King Saud University for its funding of the present research project (IRG 14-06).

\section{REFERENCES}

1. Jain PK, El-Sayed IH, El-Sayed MA (2007) Au nanoparticles target cancer. Nano Today 2, 18-29.

2. Huff TB, Tong L, Zhao Y, Hansen MN, Cheng JX, Wei A (2007) Hyperthermic effects of gold nanorods on tumor cells. Nanomedicine 2, 125-32.

3. Chen J, Wang D, Xi J, Au L, Siekkinen A, Warsen A, Li ZY, Zhang $\mathrm{H}$, et al (2007) Immuno gold nanocages with tailored optical properties for targeted photothermal destruction of cancer cells. Nano Lett 7, 1318-22.

4. Sharma P, Brown SC, Bengtsson N, Zhang Q, Walter GA, Grobmyer SR, Santra S, Jiang H, et al (2008) Gold-speckled multimodal nanoparticles for noninvasive. Chem Mater 20, 6087-94.

5. Yu LE, Yung LYL, Ong CN, Tan YL, Balasubramaniam KS, Hartono D, et al (2007) Translocation and effects of gold nanoparticles after inhalation exposure in rats. Nanotoxicology 1, 235-42.

6. Pan Y, Neuss S, Leifert A, Fischler M, Wen F, Simon U, Schmid G, Brandau W, Jahnen-Dechent W (2007) Size-dependent cytotoxicity of gold nanoparticles. Small 3, 1941-9.

7. Wang SG, Lu WT, Tovmachenko O, Rai US, Yu HT, Ray PC (2008) Challenge in understanding size and shape dependent toxicity of gold nanoparticles in human skin keratinocytes. Chem Phys Lett 463, 145-9.

8. Pissuwan D, Valenzuela SM, Cortie MB (2006) Therapeutic possibilities of plasmonically heated gold nanoparticles. Trends Biotechnol 24, 62-7.

9. Visaria R, Bischof JC, Loren M, Williams B, Ebbini E, Paciotti G, Griffin R (2007) Nanotherapeutics for enhancing thermal therapy of cancer. Int J Hyperther 23, 501-11.

10. Chithrani BD, Chan WC (2007) Elucidating the mechanism of cellular uptake and removal of protein-coated gold nanoparticles of different sizes, shapes. Nano Lett 7, 1542-50.

11. BarathManiKanth S, Kalishwaralal K, Sriram M, Pandian SRK, Youn HS, Eom SH, Gurunathan S (2010) Anti-oxidant effect of gold nanoparticles restrains hyperglycemic conditions in diabetic mice. J Nanobiotechnol 8, 16. 
12. Abdelhalim MA, Jarrar BM (2011) Gold nanoparticles administration induced prominent inflammatory, central vein intima disruption, fatty change and Kupffer cells hyperplasia. Lipids Health Dis 10, 133.

13. Abdelhalim MA, Jarrar BM (2011) The appearance of renal cells cytoplasmic degeneration and nuclear destruction might be an indication of GNPs toxicity. Lipids Health Dis 10, 147.

14. Abdelhalim MA, Jarrar BM (2011) Renal tissue alterations were-size dependent with smaller ones induced more effects and related with the exposure of gold nanoparticles. Lipids Health Dis 10, 163.

15. Yen IJ, Hsu SH, Tsai CL (2009) Cytotoxicity and immunological response of gold and silver nanoparticles of different sizes. Small 5, 1553-61.

16. Zhang XD, Di W, Shen X, Liu PX, Yang N, Zhao B, Zhang H, Sun YM, Zhang LA, Fan FY (2011) Size-dependent in vivo toxicity of PEG-coated gold nanoparticles. Int $J$ Nanomed 6, 2071-81.

17. Khalil ML (2006) Biological activity of bee propolis in health and disease. Asian Pac J Canc Prev 7, 22-31.

18. Valadares BL, Graf U, Spanó MA (2008) Inhibitory affects of water extract of propolis on doxorubicininduced somatic maturation and recombination in Drosophila melanogaster. Food Chem Toxicol 46, 1103-10.

19. Ota C, Unterkircher C, Fontinato V, Shimizu MT (2001) Antifungal activity of propoplis on different species of Candida. Mycoses 44, 375-8.

20. Almeida EC, Menezes H (2002) Anti-inflammatory activity of propolis extracts: a review. $J$ Venom Anim Toxins 8, 191-212.

21. Lee YJ, Liao PH, Chen WK, Yang CY (2000) Preferential cytotoxicity of caffiec acid phenethyl ester analogous on oral cancer cells. Canc Lett 153, 51-6.

22. Urgur A, Arslan T (2004) An in vitro study on antimicrobial activity of propolis from Mugla province of Turkey. J Med Food, 7, 90-4.

23. Lejeune B, Pourrat A, Dehmouche H (1988) Propolis use in dermatocosmetology. Parfums Cosmet Aromes 82, 73-7.

24. Shukla S, Bhadauria M, Jadon A (2005) Evaluation of hepatoprotective potential of propolis extract in carbon tetrachloride induced liver injury in rats. Indian J Biochem Biophys 42, 321-5.

25. Naik DG, Vaidya HS, Behera BC (2009) Antioxidant properties of Indian propolis. J ApiProd ApiMed Sci 1, 110-20.

26. Zhao JQ, Wen YF, Bhadauria M, Nirala SK, Sharma A, Shrivaslava S, Shukla S, Aqrawal OP, Mathur R (2009) Protective role of propolis on inorganic mercury induced oxidative stress in mice. Indian $J \operatorname{Exp}$ Biol 47, 264-9.

27. Jarrar BM, Taib NT (2008) Histocytotechnology, 3rd edn, King Saud Univ Press, Riyadh.

28. Jarrar BM, Alferah MA (2014) Renal histological alterations induced by $10 \mathrm{~nm}$ gold nanoparticles toxicity in relation with the time of exposure. Lat Am J Pharm 33, 725-30.

29. Yousef MI, Salama AF (2009) Propolis protection from reproductive toxicity caused by aluminum chloride in male rats. Food Chem Toxicol 47, 1168-75.

30. El-Mazoudy RH, Attia A, El-Shenawy NS (2011) Protective role of propolis against reproductive toxicity of chlorpyrifos in male rats. Pestic Biochem Physiol 101, 175-81.

31. Bhadauria M (2012) Combined treatment of HEDTA and propolis prevents aluminum induced toxicity in rats. Food Chem Toxicol 50, 2487-95.

32. Yousef MI, Kamel KI, Hassan MS, El-Morsy AMA (2010) Protective role of propolis against reproductive toxicity of triphenyltin in male rabbits. Food Chem Toxicol 48, 1846-52.

33. Russo A, Troncoso N, Sanchez F, Garbarino JA, Vanella A (2006) Propolis protects human spermatozoa from DNA damage caused by benzo[ $a]$ pyrene and exogenous reactive oxygen species. Life Sci 78, 1401-6.

34. Guimarães NSS, Mello JC, Paiva JS, Bueno PCP, Berretta AA, Torquato RJ, Nantes IL, Rodrigues T (2012) Baccharis dracunculifolia, the main source of green propolis, exhibits potent antioxidant activity and prevents oxidative mitochondrial damage. Food Chem Toxicol 50, 1091-7.

35. Kumar V, Abbas AK, Aster JC (2015) Robbins \& Cotran Pathologic Basis of Disease, 9th edn, SaundersElsevier, Philadelphia. 